\title{
Quality Management in Medical Laboratories for Accreditation
}

\author{
Rayat CS* \\ Department of Histopathology, Postgraduate Institute of \\ Medical Education \& Research, India \\ *Corresponding author: Charan Singh Rayat, \\ Department of Histopathology, Postgraduate Institute \\ of Medical Education \& Research, Chandigarh-160012, \\ India
}

Received: March 20, 2017; Accepted: March 27, 2017; Published: April 03, 2017

\section{Editorial}

Quality assurance in medical laboratories requires a great deal of compliance of an International Standard or a National Standard for quality and competence. A medical laboratory or clinical laboratory is a laboratory for the examination of materials derived from the human body for a purpose of providing necessary information for diagnosis, prevention and treatment of disease as well as for follow up evaluation of health of a patient during the treatment of an ailment. The investigations could be biological, hematological, immunohematological, biochemical, microbiological, immunological, biophysical, cytological, histological, immunopathological, molecular or genetic. Modern medicine warrants the thorough investigations of biological material derived from the body of a patient to ascertain the cause and effect of disease before the commencement of medical or surgical treatment. Various laboratory investigations and interpretations of results provide advisory service to the consultant physician and/or surgeon. The 'International Organization for Standardization' (ISO) is an 'International Authority' for setting up standard guidelines for various organizations and laboratories. The International Organization for Standardization is based in Geneva, Switzerland and 163 countries are its members. The ISO collaborates closely with the 'International Electrotechnical Commission (IEC) on all matters of 'Electrotechnical Standardization'. The ISO 15189 was compiled by Technical Committee ISO/TC-212, for 'Clinical laboratory testing and in vitro diagnostic test systems'. The International Standard for Medical Laboratories was first published in the year 2005 based on ISO 15189:2003 ( $1^{\text {st }}$ Edition) and later revised as ISO 15189:2007 ( $2^{\text {nd }}$ Edition). Further revision was done in the year 2010 and the current version ISO 15189:2012 ( $3^{\text {rd }}$ Edition) [1] provides standard guidelines for 'Requirements for Quality and Competence in Medical Laboratories'. The ISO 15189:2012 is based upon ISO/IEC 17025:2005 [2] and ISO 9001.

The main goal of ISO 15189:2012 is the 'Global Harmonization of Medical Laboratories' in terms of quality of their services. A medical laboratory's fulfilment of the requirements of standard (both the technical competence requirements as well as quality management system requirements) means that the laboratory could be accredited for consistency in technically valid results. Bodies of National Standards of various countries have been adapting ISO
15189:2012 for regulating requirements of quality and competence in medical laboratories. The quality policy in medical laboratories has been adapted from ISO 9000:2005 [3]. The ISO 9000 family of standards describes the fundamentals of quality management systems and specifies the terminology for quality management systems. The ISO 9001 specifies requirements for a quality management system where an organization needs to exhibit its ability to provide products or services that fulfill customer as well as applicable regulatory requirements [4]. The organization would also aim to enhance customer satisfaction. The term quality has its inherent characteristics like poor, good or excellent quality. The fulfillment of requirements of ISO Standard would assure the excellent quality.

The 'College of American Pathologists' (CAP) is an 'Accreditation Authority' approved by the 'Centres for Medicare and Medicaid Services' (CMS) in the United States of America (USA) [5]. CAP$15189^{\mathrm{SM}}$ is a voluntary non-regulatory accreditation to the 'ISO 15189:2012 Standard' as published by ISO. The CAP $15189^{\mathrm{SM}}$ does not replace CAP's 'Clinical Laboratory Improvement Amendments' (CLIA) based 'Laboratory Accreditation Program', but complements CAP accreditation as well as other quality systems. The accreditation program optimizes the processes to improve patient care, strengthens quality standards while reducing institutional errors and risks as well as controls cost of investigations. The CAP- $15189^{\mathrm{SM}}$ is also an educational program that offers a highly disciplined approach to implementing and sustaining quality assurance parameters. The laboratories certified under the standard module also work towards continual improvement, recognize their problems and seek intelligent solutions, thereby enhancing patient care as well as customer and staff satisfaction.

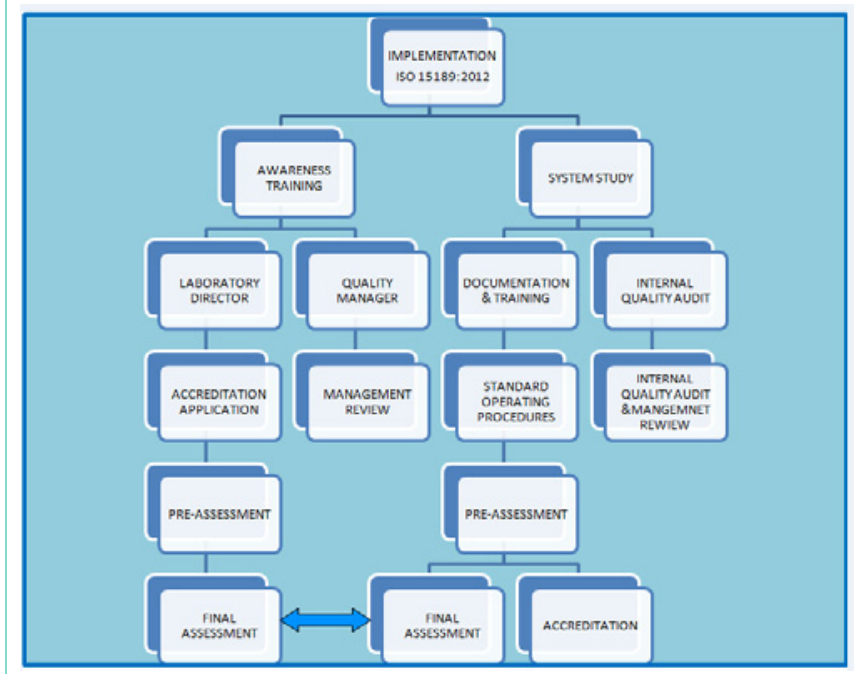

Figure 1: Schematic illustration on road map to implementation of ISO 15189:2012. 


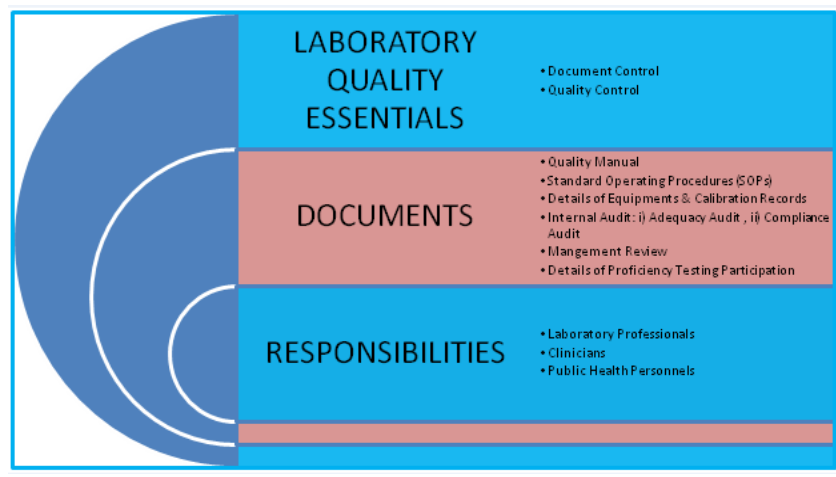

Figure 2: Quality essentials for a medical laboratory and their interrelationship.

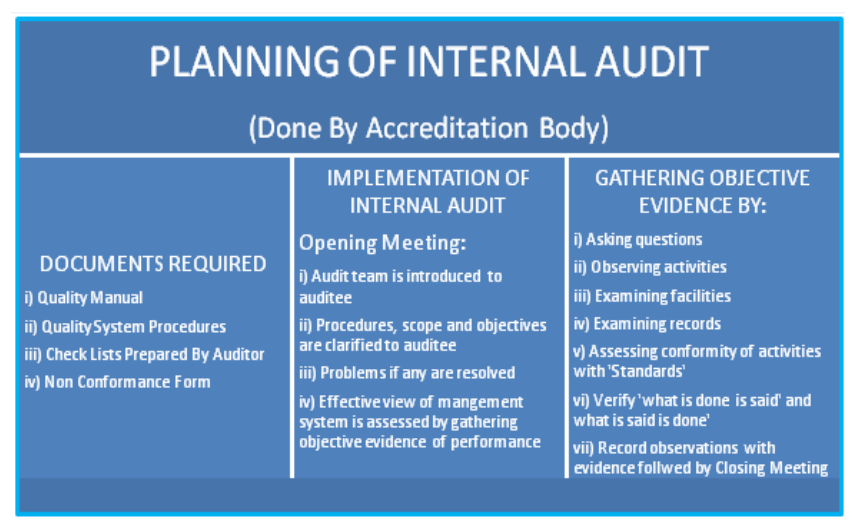

Figure 3: Flow diagram illustrating the systematic planning of internal audit.

Let us have a look at the scope and fundamentals of ISO 15189:2012. Scope: This International Standard can be used by the medical/clinical laboratories in developing their quality assurance systems as well as assessing their own competence. It can also be used for conformity assessment or recognizing the competence of medical laboratories by regulating authorities, laboratory customers and accreditation bodies. Terms and definitions: The procedure by which an authority gives formal recognition that an organization or medical/ clinical laboratory is competent to carry out specific tasks is termed as 'accreditation'. The scheduled time frame/interval about examination results for an alert test indicating risk to the patient of injury or death is termed as 'alert interval/critical interval'. The process by which patient's examination results are sent to 'Laboratory Information System' (LIS) and compared with laboratory defined reference values automatically and included in the patient report formats is termed as 'automated selection and reporting of results'. The specified interval of the distribution of values taken from a biological reference population is termed as 'biological reference interval' [6]. The demonstrated ability to apply knowledge and expertise/skill for assuring quality is called 'competence'. The documented, implemented and maintained way to carry out a specific activity or process has been termed as 'documented procedure'. The measure/extent of degree to which a set of inherent characteristics fulfils the desired requirements is termed as 'quality indicator'.

The management system poised to direct and control an organization in terms of quality has been termed as 'Quality

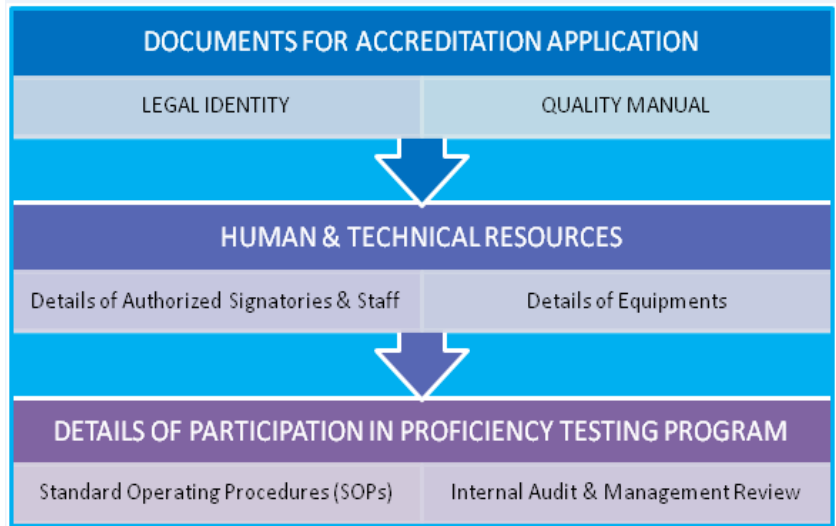

Figure 4: Depiction of seven mandatory documents required for making an application for accreditation of a Medical laboratory.

management system'. The roots of quality management system are 'quality policy' and 'quality objectives'. Overall intensions and direction of a laboratory management in relation to quality is termed as 'quality policy' and something aimed or sought to be delivered with quality has been termed as 'quality objective'.

Sections 4 and 5 of the ISO 15189:2012 deliberate on the 'Management Requirements' and 'Technical Requirements' respectively. Management Requirements are: i) Organization and management responsibility, ii) Quality management system, iii) Document control, iv) Service agreement, v) Examination of referral laboratories, vi) External services and supplies, vii) Advisory services, viii) Resolution of Complaints, ix) Identification and control of nonconformities, $\mathrm{x}$ ) Corrective action, xi) Preventive action, xii) Continual improvement, xiii) Control of records, xiv) Evaluation and audit, and xv) Management reviews. Technical Requirements are: i) Personnel, ii) Accommodation and environmental conditions, iii) Laboratory equipment, reagents and consumables, iv) Preexamination process, v) Examination process, vi) Ensuring quality of examination results, vii) Post examination process, viii) Reporting results, ix) Release of reports, and x) Laboratory information system. The scope of implementation of ISO 15189:2012 has been illustrated in (Figure 1).

This article has been carved out to impress upon awareness about quality management and recognition by national quality control authority and accreditation bodies. Whether you decide or do not decide to consider accreditation of your organization or medical laboratory, but you should follow the recommendations contained in ISO 15189:2012; as it is the right choice to do. The route to 'accreditation' is through the quality management system and delivery of quality to the customers. Let us prepare ourselves for achieving the goal.

Pre-Requisites for Laboratory: i) Legal Registration, ii) Adequate facilities, iii) Technically competent qualified staff, iv) Quality manager should have attended 'Internal Audit Training Program, v) Comply with all the requirements of ISO 15189 with reference to specific criteria, vi) Equipment calibration and traceability national/ international standard, vii) Management review, viii) Have internal quality plan and participate in 'Proficiency Testing Program' conducted by National Accreditation Body/Board. The 'Laboratory 
Quality Essentials' and their inter-relationship have been exhibited in (Figure 2). The internal audit is very essential for adequacy and technical assessments. The systematic planning of internal audit has been illustrated in (Figure 3). The seven mandatory documents (Legal identity, Quality manual, Details of authorized signatories \& staff, Details of equipments, Standard operating procedures, Internal audit and Management review) required as enclosures for making an application for accreditation of a medical laboratory have been depicted in (Figure 4).

The competence for accreditation is a worldwide obsession which sensitize us about customer centric quality culture. Every laboratory management should review the 'quality management system' at regular intervals to ensure its adequacy, continuing suitability, effectiveness and support of patient cause. Laboratory should ensure confidentiality of user data and feedback inputs. The organization or laboratory management should document qualifications, experience and skills for each position of its staff. All laboratory personnel making judgements on the results of laboratory investigations should have theoretical as well as practical experience and expertise of the subject.

The laboratory should have information available for patients and users regarding laboratory services offered, location, working hours and turnaround time. There should be clear policy and information regarding examinations referred to other laboratories. The laboratory should participate in interlaboratory comparison programs for all the examinations for self-assessment of quality. The laboratory should monitor the results of 'External Quality Assessment Systems' (EQAS) and go for corrective actions. The laboratory should use quality control materials specified for the examination system and all quality control materials should be periodically examined for stability of the procedure to avoid any risk to the patient from erroneous results. The laboratory should have procedure to prevent the release of results to patients in the event of quality control failure.

The ISO 15189:2012 is not merely intended for accreditation or certification, but a medical laboratory's fulfilment of the requirements of the standard means for both the technical competence and management system requirements for continuous delivery of technically valid results. The compliance of standard focuses us on patient safety and accuracy of test results. The standard addresses the ethics and information system needs of the medical laboratory with poise for worldwide recognition.

\section{References}

1. ISO 15189:2012. Medical Laboratories - Requirements for quality and competence.

2. ISO/IEC 17025:2005. General requirements for the competence of testing and calibration laboratories.

3. ISO 9000:2005. Quality management systems - Fundamentals and vocabulary.

4. ISO 9001:2008. Quality management systems - Requirements.

5. College of American Pathologists. Quality management in clinical laboratories, CAP, Northfield, IL. 2005.

6. Solberg HE, Burtis CA, Ashwood ER. Establishment and use of reference values, Tietz Textbook of Clinical Chemistry and Molecular Diagnostics. St Louis, Missouri: Elsevier Saunders. 2005.
Austin J Pathol Lab Med - Volume 4 Issue 1 - 2017 ISSN : 2471-0156 | www.austinpublishing group.com Rayat. (C) All rights are reserved
Citation: Rayat CS. Quality Management in Medical Laboratories for Accreditation. Austin J Pathol Lab Med. 2017; 4(1): 1019 